\title{
Static Decoupling in fault detection
}

\section{Niemann, Hans Henrik}

Published in:

Decision and Control, 1998. Proceedings of the 37th IEEE Conference on

Link to article, DOI:

10.1109/CDC.1998.760850

Publication date:

1998

Document Version

Publisher's PDF, also known as Version of record

Link back to DTU Orbit

Citation (APA):

Niemann, H. H. (1998). Static Decoupling in fault detection. In Decision and Control, 1998. Proceedings of the 37th IEEE Conference on (Vol. 1, pp. 1131-1136). IEEE. https://doi.org/10.1109/CDC.1998.760850

\section{General rights}

Copyright and moral rights for the publications made accessible in the public portal are retained by the authors and/or other copyright owners and it is a condition of accessing publications that users recognise and abide by the legal requirements associated with these rights.

- Users may download and print one copy of any publication from the public portal for the purpose of private study or research.

- You may not further distribute the material or use it for any profit-making activity or commercial gain

- You may freely distribute the URL identifying the publication in the public portal

If you believe that this document breaches copyright please contact us providing details, and we will remove access to the work immediately and investigate your claim 


\title{
Static Decoupling in Fault Detection
}

\author{
Henrik Niemann* \\ Department of Automation, Technical University of Denmark \\ Building 326, DK-2800 Lyngby, Denmark \\ E-mail: hhn@iau.dtu.dk
}

\begin{abstract}
An algebraic approach is given for a design of a static residual weighting factor in connection with fault detection. A complete parameterization is given of the weighting factor which will minimize a given performance index.
\end{abstract}

\section{Introduction}

The increasing use of supervisors in connection with control make it necessary to include fault detectors in the control architecture. The fault detectors are used for detection fault in dynamic systems which cannot be allow in the feedback control. When a fault is detected, it is then the supervisor unit that need to take care of this fault situation by e.g. close down the system or by change controller etc. A lot of different types of fault can appear in a dynamic system, as e.g. actuator and sensor faults, slowly changes of system parameters etc. The condition to obtain a complete reliable control architecture is that all elements will work reliable. So we need to have reliable detection of the faults in the system.

The design of fault detectors includes both a design of a filter for the detection and also a selection of a threshold value for the filter, see e.g. [9]. The selection of the threshold is very important. If the threshold is selected too high, a number of faults will not be detected. On the other hand, if the threshold is selected too small, we will get a number of false alarms due to disturbance. None of the cases are in general

\footnotetext{
*Supported by the Danish Technical Research Council under grant no. 96-01557.
}

useful when we want a reliable fault detection. To optimize the fault detection, the filter and the threshold value need to be considered at the same time and not as two separate designs. However, the design of a detection filter and the selection of the threshold is normally considered separately. In e.g. [12], the two part are connected for optimization of the smallest fault signal that can be detected. An implicit design method has been given in [12].

There exist a number of different way to design fault detectors, see e.g. [9] and [10]. One way to design a fault detector is based on residual vectors. This mean that we will not get a direct estimation of the fault signal, but an residual vector which must be small when no fault appear in the system and large, in some sense, when faults appear in the system. A residual vector consist of a filter/observer and a residual weighting matrix. The residual weighting matrix can both be a constant matrix or dynamic. Further, the design of the filter and the weight matrix can be done in one step, see [8], or it can be done in two steps, see e.g. [11].

The motivation for using residual vectors instead of using estimates of the fault signals directly is that we do not in general need to know the faults exactly. In general we are satisfied by knowing that, at first, that there are faults in the system (fault detection) and, at second, which faults that has appear in the system (fault isolation). In the cases where we can accept some minor faults, we just need to select the threshold value such that these fault signals are not detected.

In this paper we will only consider the design of static residual weighting matrices. In [11], the 
eigenstructure assignment method has been applied for the design of an observer and a static residual weighting matrix. The main idea in this approach is to design the observer gain and the weight matrix such that the residual vector is complete decoupled from disturbance input. Conditions are given for obtaining this. This mean that not all fault vectors can be detected. The result derived in this paper give a complete parameterization of the residual weighting matrix of both all residual vectors that are complete decoupled from the disturbance input as well as a parameterization of all residual vectors that can not be decoupled from the disturbance input in steady state. Further, a complete description of which fault vectors that can be detected disturbance free and which cannot be detected disturbance free in the steady state case is given.

It need to be pointed out in this connection that the main reason for making exact decoupling is that we can use zero as the threshold value. This will take care of the problem with non detected faults and false alarms. However, it is not all fault signals that can be detected disturbence free.

\section{Problem Formulation}

The FDI design setup will be given in the following. Consider the following system $G$ given by:

$$
y=G_{y w}(s) w+G_{y f}(s) f
$$

where $w \in \mathcal{R}^{m}$ is a disturbance signal vector, $f \in \mathcal{R}^{p}$ is a fault signal vector and $y \in \mathcal{R}^{q}$ is the measurement output vector. Further, it is assumed that $q \geq p>m$ and that none disturbance inputs are identical with any fault inputs at the system.

A filter is now applied to estimate the fault signal vector $f$ out from the measurement signal vector $y$. Let the filter be given by $F(s)$, i.e. the estimate of the fault signal vector is given by:

$$
\hat{f}=F(s)\left(G_{y w}(s) w+G_{y f}(s) f\right)
$$

The design of $F(s)$ will not be considered in this paper, see instead e.g. [11], [10], [7], [4] and
[8] for mention a few. It will only be assumed that the rank of $F G_{y f}$ in steady state is maximal.

Further, let's consider the residual vector for the fault detection in (2) given by:

$$
r=R F(s)\left(G_{y w}(s) w+G_{y f}(s) f\right)
$$

where $R$ is the residual weighting matrix. This matrix can be dynamic but will in most cases be a constant matrix. $R$ will be a constant matrix in the following.

Before the problem for the design of the residual weighting matrix is given, we need to consider a performance index for the fault detection problem. Different performance indices for fault detection has been considered in a number of paper, see e.g. [5], [13], [2], [3] and [12]. From [3] we have the following performance index for the residual vector in (3):

$$
J=2 \inf _{R} \frac{\left\|R F G_{y w}\right\|}{\left\|R F G_{y f}\right\|_{-}}
$$

where $\|M\|=\sup _{\|x\|}\|M x\|$ and $\|M\|_{-}=$ in $f_{\|x\|}\|M x\|_{e}$, where $\|\cdot\|_{e}$ is an evaluation function, which may not be a norm.

This index is not so useful in connection with design of the fault detector $F(s)$. This has been discussed in [12], where a new performance index has been derived only based on norms, which is not the case with the index given by (4). However, for the optimization of the static residual weighting matrix $R$, the performance index in (4) can almost be used directly. The only modification we will do is to make a separation of the index and use different threshold values for every single residual signal. An interpretation of the index given in (4) is that it gives the norm of the smallest fault signal that is guarantee to be detected. The index is given as the largest gain from disturbance to residual vector divided by the smallest gain from fault signal to residual vector. This might be conservative due to the fact that the maximal gain from disturbance to residual vector will in general not be in the same direction as the minimal gain from fault signal to residual vector. In the general case when only a single residual signal is considered, we can give 
the following performance index:

$$
J_{i}=2 \inf _{R} \frac{\left\|g_{w, i}\right\|}{\left\|R F G_{y f}\right\|_{-}}, \quad i=1, \ldots, p
$$

where $g_{w, i}$ appear from a partition of $R F G_{y w}$ given by:

$$
R F G_{y w}=\left[\begin{array}{c}
g_{w, 1} \\
\vdots \\
g_{w, i} \\
\vdots \\
g_{w, p}
\end{array}\right]
$$

It should be mentioned in this connection that this new performance index $J_{i}$ in (5) is still potential conservative. This new index is just an straightforward extension of the index given in (4) to the case where the single residual signals are considered separately. Again, it can be seen directly from (5) that we need to reduce the transfer function from the disturbance to the residual vectors given by $g_{w, i}$ for minimizing the performance index $J_{i}$. The optimal is to make these transfer function equal to zero, i.e. exact decoupling of disturbance in the residuals. However, this will not in general be possible to decouple the disturbance exactly from all residual signals. When this is not possible, let's consider the case when a reduced number of residuals are exactly decoupled from the disturbance input. We are now able to formulate the following static optimization problem.

Problem 1 Let the residual vector be given by (3). Design the static residual weighting matrix $R$ such that the number of performance indices $J_{i}, i=1, \ldots, p$ satisfying

$$
J_{i}(0)=0
$$

in steady state is optimized.

The steady state detection is important in many cases as pointed out in e.g. [1]. Further, if we have obtained a static decoupling, we will in many cases also have a good dynamic decoupling. Another thing is that the fault detector will no increase in order when we only use static residual weighting matrices, which is also important in some cases.

\section{$3 \quad$ Static Decoupling}

The static design problem given in Problem 1 will be considered in this section. Let the residual vector $r$ be described by the following equation in the steady state case:

$$
r=R\left(H_{w} w+H_{f} f\right)
$$

where $H_{w}=F G_{y w}(0)$ and $H_{f}=F G_{y f}(0)$. Further, let the rank of $H_{w}$ is given by $\operatorname{rank}\left(H_{w}\right)=$ $s \leq m$.

A singular value decomposition of $H_{w}$ is given by

$$
H_{w}=U \Sigma V^{T}
$$

where $U$ and $V$ satisfies $U U^{T}=U^{T} U=I$, $V V^{T}=V^{T} V=I$ and $\Sigma$ has a canonical structure

$$
\Sigma=\left[\begin{array}{cc}
\Sigma_{0} & 0 \\
0 & 0
\end{array}\right], \quad \Sigma_{0}=\operatorname{diag}\left(\sigma_{1}, \cdots, \sigma_{s}\right)>0
$$

Based on this singular value decomposition of $H_{w}$, all residual weighting matrices can then be given by:

$$
\begin{aligned}
R= & Z\left(I-H_{w} H_{w}^{+}\right)+X H_{w} H_{w}^{+} \\
= & Z\left(I-U\left[\begin{array}{cc}
I_{s} & 0 \\
0 & 0
\end{array}\right] U^{T}\right) \\
& +X U\left[\begin{array}{cc}
I_{s} & 0 \\
0 & 0
\end{array}\right] U^{T}
\end{aligned}
$$

where $X$ and $Z$ are two arbitrary $p \times q$ matrices and $H_{w}^{+}$is the Moore-Penrose inverse of $H_{w}$.

Now let $Z$ and $X$ be given by

$$
X=\bar{X} U^{T}, Z=\bar{Z} U^{T}
$$

(8) can be rewritten into

$$
R=\bar{Z}\left[\begin{array}{cc}
0 & 0 \\
0 & I_{q-s}
\end{array}\right] U^{T}+\bar{X}\left[\begin{array}{cc}
I_{s} & 0 \\
0 & 0
\end{array}\right] U^{T}
$$

Further let $\bar{X}$ and $\bar{Z}$ be partitioned as

$$
\bar{X}=\left[\begin{array}{ll}
X_{1} & X_{2}
\end{array}\right], \bar{Z}=\left[\begin{array}{ll}
Z_{1} & Z_{2}
\end{array}\right]
$$

where $X_{1}, Z_{1} \in \mathcal{R}^{p \times s}, X_{2}, Z_{2} \in \mathcal{R}^{p \times(q-s)}$. Then all $R$ can be described by

$$
R=\left[\begin{array}{ll}
X_{1} & Z_{2}
\end{array}\right] U^{T}
$$


With this parameterization of $R$ given by (9), it is simple to see that

$$
R H_{w}=0, \text { for } X_{1}=0
$$

which give an explicit parameterization of all residual weighting matrices that satisfies $R H_{w}=$ 0 .

Before this residual weighting matrix is used in (6), let $\left[\begin{array}{ll}X_{1} & Z_{2}\end{array}\right]$ be partitioned as

$$
\left[\begin{array}{ll}
X_{1} & Z_{2}
\end{array}\right]=\left[\begin{array}{ll}
X_{11} & Z_{21} \\
X_{12} & Z_{22}
\end{array}\right]
$$

where $X_{11} \in \mathcal{R}^{s \times s}, X_{12} \in \mathcal{R}^{(p-s) \times s}, Z_{21} \in$ $\mathcal{R}^{s \times(q-s)}$ and $Z_{22} \in \mathcal{R}^{(p-s) \times(q-s)}$.

Using the above $R$ matrix in (8) in the equation for the residual vector, we get:

$$
\begin{aligned}
r= & R\left(H_{w} w+H_{f} f\right) \\
= & {\left[\begin{array}{ll}
X_{11} & Z_{21} \\
X_{12} & Z_{22}
\end{array}\right] U^{T}\left(H_{w} w+H_{f} f\right) } \\
= & {\left[\begin{array}{ll}
X_{11} \Sigma_{0} & 0 \\
X_{12} \Sigma_{0} & 0
\end{array}\right] V^{T} w } \\
& +\left[\begin{array}{ll}
X_{11} & Z_{21} \\
X_{12} & Z_{22}
\end{array}\right] U^{T} H_{f} f
\end{aligned}
$$

It can be seen directly from the above equation that we need to select $X_{12}=0$ to decouple the last $p-s$ residual signals complete from the disturbance input $w$. The residual vector is then given by

$r=\left[\begin{array}{cc}X_{11} \Sigma_{0} & 0 \\ 0 & 0\end{array}\right] V^{T} w+\left[\begin{array}{cc}X_{11} & Z_{21} \\ 0 & Z_{22}\end{array}\right] U^{T} H_{f} f$

Moreover, it might be possible to decouple more residual vectors from the disturbance. This is possible when

$$
\operatorname{rank}\left[H_{w} H_{f}\right]=t>p
$$

It is then possible to make further $t-p$ residual signals disturbance free. To detect and isolate all $p$ fault signals, it is required that the rank of $R$ is $p$. When it is assumed that $Z_{22}$ has full rank, $p-s$. Let $Z_{21}$ be partitioned as follows

$$
Z_{21}=\left[\begin{array}{l}
Z_{21,1} \\
Z_{21,2}
\end{array}\right]
$$

where $Z_{21,1} \in \mathcal{R}^{(s-t+p) \times(q-s)}$ and $Z_{21,2} \in$ $\mathcal{R}^{(t-p) \times(q-s)}$. Now, let $Z_{21,2}$ be selected such that

$$
\operatorname{rank}\left[\begin{array}{c}
Z_{21,2} \\
Z_{22}
\end{array}\right]=t-s
$$

Now let $X_{1}$ be partitioned as follows:

$$
X_{11}=\left[\begin{array}{l}
X_{11,1} \\
X_{11,2}
\end{array}\right]
$$

where $X_{11,1} \in \mathcal{R}^{(s-t+p) \times(q-s)}$ and $X_{11,2} \in$ $\mathcal{R}^{(t-p) \times(q-s)}$. We can now select $X_{11,2}=0$ and then make $t-p$ residual vectors exact decoupled from the disturbance inputs. The residual vector is then given by:

$$
\begin{aligned}
r= & {\left[\begin{array}{cc}
X_{11,1} \Sigma_{0} & 0 \\
0 & 0 \\
0 & 0
\end{array}\right] V^{T} w } \\
& +\left[\begin{array}{cc}
X_{11,1} & Z_{21,1} \\
0 & Z_{21,2} \\
0 & Z_{22}
\end{array}\right] U^{T} H_{f} f
\end{aligned}
$$

Let $U$ and $V$ be partitioned as follows

$$
\begin{aligned}
U & =\left[\begin{array}{ll}
U_{1} & U_{2}
\end{array}\right] \\
V & =\left[\begin{array}{ll}
V_{1} & V_{2}
\end{array}\right]
\end{aligned}
$$

and let

$$
\begin{aligned}
\tilde{w} & =V_{1}^{T} w \\
\bar{X}_{11,1} & =X_{11,1} \Sigma_{0}
\end{aligned}
$$

It is now possible to modify the residual vector into

$$
\begin{aligned}
r= & {\left[\begin{array}{c}
\bar{X}_{11,1} \\
0 \\
0
\end{array}\right] \tilde{w} } \\
& +\left[\begin{array}{c}
\bar{X}_{11,1} \Sigma_{0}^{-1} U_{1}^{T}+Z_{21,1} U_{2}^{T} \\
Z_{21,2} U_{2}^{T} \\
Z_{22} U_{2}^{T}
\end{array}\right] H_{f} f
\end{aligned}
$$

Based on the above derivation, we have then the following result.

Theorem 1 Let the residual vector be defined by (3). Further, let the static residual weighting matrix $R$ be given by

$$
R=\left[\begin{array}{c}
\bar{X}_{11,1} \Sigma_{0}^{-1} U_{1}^{T}+Z_{21,1} U_{2}^{T} \\
Z_{21,2} U_{2}^{T} \\
Z_{22} U_{2}^{T}
\end{array}\right]
$$


where $\bar{X}_{11,1} \in \mathcal{R}^{(s-t+p) \times(q-s)}, \quad Z_{21,1} \in$ $\mathcal{R}^{(s-t+p) \times(q-s)}, Z_{21,2} \in \mathcal{R}^{(t-p) \times(q-s)}$ and $\bar{Z}_{22} \in$ $\mathcal{R}^{(p-s) \times(q-s)}$.

Assume that $\bar{X}_{11,1}$ is selected such that the rows has a norm equal to 1 and that $Z_{21,2}$ and $Z_{22}$ are selected such that:

$$
\operatorname{rank}\left[\begin{array}{c}
Z_{21,2} \\
Z_{22}
\end{array}\right]=t-s
$$

Then the $p$ performance indices given by (5) are then given by:

$$
J_{i}=0
$$

for $i=1, \cdots, t-s$ and

$$
J_{i}=\frac{2}{\left\|\left[\begin{array}{c}
\bar{X}_{11,1} \Sigma_{0}^{-1} U_{1}^{T}+Z_{21,1} U_{2}^{T} \\
Z_{21,2} U_{2}^{T} \\
Z_{22} U_{2}^{T}
\end{array}\right] H_{f}\right\|_{-}}
$$

for $i=t-s+1, \cdots, p$ in steady state.

Based on Theorem 1, get get directly the following lemma.

\section{Lemma 2 If}

$$
\operatorname{rank}\left[H_{w} H_{f}\right]=\operatorname{rank}\left[H_{w}\right]+\operatorname{rank}\left[H_{f}\right]
$$

then the $p$ performance indices are given by

$$
J_{i}=0, i=1, \cdots, p
$$

This result is equivalent with the results given in [6] for the dynamic case.

The results in Theorem 1 and Lemma 2 are discussed in next section.

\section{Interpretation of the Decou- pling Result}

The main emphasis of Theorem 1 is that it is possible to decouple disturbance exactly from some residual vectors. This mean that we can use 0 as the threshold value for some residual signals without obtaining any false alarms in steady state. More precise, the dimension of the space $S_{0}$ given by the span of fault vectors that are decoupled from the disturbance is $t-s$. Further the space $S_{0}$ is given by:

$$
S_{0}=\operatorname{span}\left(v_{f, p-t+s+1}, \cdots, v_{f, p}\right)
$$

where $v_{f, i}$ are the vectors defined by:

$$
V_{f}=\left[v_{f, 1}, \cdots, v_{f, s}\right]
$$

where $V_{f}$ is a unitary matrix satisfying the singular value decomposition of $U_{2}^{T} H_{f}$ :

$$
U_{2}^{T} H_{f}=U_{f} \Sigma_{f} V_{f}^{T}
$$

Moreover the space $S_{1}$ including the fault vectors which can not be detected disturbance free is given by:

$$
S_{1}=S_{0}^{\perp}=\operatorname{span}\left(v_{f, 1}, \cdots, v_{f, p+s-t}\right)
$$

By selecting the arbitrary matrices $\bar{X}_{11,1}, Z_{21,2}$ and $Z_{22}$ in Theorem 1 so they have full rank, then the span of all possible residual vectors will be of dimension $p$. This mean that it is also possible to make fault isolation by using the residual vector $r$.

The most common case in fault detection is when only one fault appear, i.e. $f=e_{i}, i=$ $1, \cdots, p$ where $e_{i}$ is the i'th unit vector. The condition for detection a single fault without any disturbance is given in the following lemma.

Lemma 3 Let the residual vector be given by (11). The single fault $f=e_{i}, i=1, \cdots, p$ is detected disturbance free if and only if

$$
r_{2}=\left[\begin{array}{c}
Z_{21,2} \\
Z_{22}
\end{array}\right] U_{2}^{T} H_{f} f \neq 0
$$

It is easy to see that the i'th fault $f=e_{i}$ is detected if the $i$ 'th column of $\left[\begin{array}{c}Z_{21,2} \\ Z_{22}\end{array}\right] U_{2}^{T} H_{f}$ is non zero.

The free matrix $X_{11,1}$ in Theorem 1 must be selected such that the last $p-t+s$ indices $J_{i}$ are minimized. This matrix can be optimized by e.g. using an optimization toolbox.

The result given in Lemma 2 give the case where the number of measurements is equal to the number of fault signals and the number of 
disturbance signals that are not dynamic decoupled from the fault estimation signals. Further it is assumed that no disturbance and fault signal has the same input matrix. In this case, it is possible to make exact disturbance decoupling in steady state.

In the beginning of Section 2, it was assumed that no disturbance inputs and fault inputs enter the system at the same input. This might not always be the case. If this condition is not satisfied, we can still use the above static disturbance decoupling method. In this case, it will not be possible to detect the fault signals, which enter the system at the same inputs as disturbance, in the disturbance free residual vector $r_{2}$.

\section{References}

[1] J. Chen, R.J. Patton, and G.P. Liu. Optimal residual design for fault diagnosis using multi-objective optimization and genetic algorithms. International $J$. of System Science, 27:567-576, 1996.

[2] X. Ding and P.M. Frank. Frequency domain approach and threshold selector for robust model-based fault detection and isolation. In Proceedings of IFAC Symposium SAFEPROCESS'91, pages 307-312, Barden-Barden, Germany, 1991.

[3] X. Ding and L. Guo. Observer based optimal fault detector. In Proceedings of the 13th IFAC World Congress, volume N, pages 187-192, San Francisco, CA, USA, 1996.

[4] A. Edelmayer, J. Bokor, and L. Keviczky. An $\mathcal{H}_{\infty}$ filtering approach to robust detection of failures in dynamical systems. In Proceedings of the 33rd Conference on Decision and Control, pages 3037-3039, Lake Buena Vista, FL, USA, 1994.

[5] A. Emami-Naeini, M.M. Akhter, and S.M. Rock. Effect of model uncertainty on failure detection: The threshold selector. IEEE Transactions on Automatic Control, 33:1106-1115, 1988.
[6] P.M. Frank and X. Ding. Frequency domain approach to optimally robust residual generation and evaluation for model-based fault diagnosis. Automatica, 30:789-804, 1994.

[7] R.S. Mangoubi, B.D. Appleby, G.C. Verghese, and W.E. VanderVelde. A robust failure detection and isolation algorithm. In Proceedings of the 34th Conference on Decision and Control, pages 2377-2382, New Orleans, LA, USA, 1995.

[8] H.H. Niemann and J. Stoustrup. Filter design for failure detection and isolation in the presence of modeling errors and disturbances. In Proceedings of the 35th IEEE Conference on Decision and Control, pages 1155-1160, Kobe, Japan, 1996.

[9] R. Patton. Robust model-based fault diagnosis: The state of art. In Proceedings IFAC Symp. SAFEPROCESS '94, pages 1-24, Espoo, Finland, 1994.

[10] R. Patton, P. Frank, and R. Clark. Fault diagnosis in dynamic systems - Theory and application. Prentice Hall, 1989.

[11] R.J. Patton and J. Chen. Robust fault detection using eigenstructure assignment: A tutorial consideration and some new results. In Proceedings of the 30th Conference on Decision and Control, pages 2242-2247, Brighton, England, 1991.

[12] M.L. Rank and H.H. Niemann. Norm based design of fault detectors. submitted for publication, 1997.

[13] J.L. Weiss. Threshold computations for detection of failures in siso systems with transfer function errors. In Proceedings of the American Control Conference, pages 22132218, Atlanta, GR, USA, 1993. 